\title{
Autorregulación del aprendizaje en centros educativos de Granada donde se utilizan las Tecnologías de la Información y la Comunicación
}

\author{
Carolina Barría Lobos. Universidad de Huelva \\ Sonia Rodríguez Fernández. Universidad de Granada \\ Purificación Salmerón Vílchez. Universidad de Granada
}

Recepción: 6 de marzo de 2017 | Aceptado: 20 de marzo de 2017
Correspondencia: Carolina Barría Lobos | Correo-e: cbarrialobos@gmail.com

iD 0000-0002-0078-3434

Citar: Barría López, C., Rodríguez Fernández, S. y Salmerón Vílchez, P. (2017). Autorregulación del aprendizaje en centros educativos de Granada donde se utilizan las tecnologías de la información y la comunicación. ReiDoCrea, 6, 140-155.

Resumen: Este trabajo tiene como objetivo analizar la utilización de estrategias de aprendizaje autorregulado en el proceso de aprendizaje de los estudiantes antes, durante y al finalizar una tarea, la percepción que manifiesta el profesorado sobre el empleo que le da el alumnado a éstas y la relación existente entre ambas opiniones, en contextos educativos en los que se utilizan las TIC. El estudio se encuentra basado en el marco teórico propuesto por Pintrich (2000) sobre el aprendizaje autorregulado. Los datos fueron obtenidos a través de un autoinforme aplicado a 332 estudiantes de 5to y 6 to de primaria y un registro de observación aplicado a 18 profesores tutores de esos mismos niveles en siete centros educativos de Granada capital, España. De acuerdo a los resultados obtenidos, podemos determinar que son las estrategias de tipo emocional/afectivas las que más utilizan los estudiantes y que existe bastante coincidencia entre la opinión del alumnado y el profesorado.

Palabras clave: Autorregulación | TIC

\section{Self-regulation of Learning in Schools of Granada where Technologies of Information and Communication Are Applied}

\begin{abstract}
The objective of this article is to analyze the application of self-regulated learning strategies in the learning process of students before, during and after finishing a task, the perception that the teachers have about the students' use of these strategies and the relation between both their opinions in educational contexts where TIC are applied. The study is based on the theory proposed by Pintrich (2000) about self-regulated learning. The statistics were obtained by way of individual questionnaires given to 332 students from the 5th and 6th grades and a record of observations made by 18 teachers from these same grades in 7 elementary schools in the city of Granada, Spain. Based on the results, one can conclude that the emotional/affective learning strategies are the most used by the students and that there is considerable agreement between the opinions of students and teachers.
\end{abstract}

Keywords: Self-regulation | ICT

\section{Introducción}

Nuestro trabajo parte de la necesidad de conocer cómo se están desarrollando los procesos de aprendizaje en los contextos educativos en los que se utilizan las tecnologías de la información y la comunicación en las actividades educativas diarias. Específicamente nos interesamos por conocer la existencia de un proceso de aprendizaje que esté siendo autorregulado por el propio alumnado. Nos centramos en ambientes educativos que utilicen las tecnologías para la labor docente diaria puesto que en los últimos años ha habido un notable incremento de dichos medios en las aulas.

Para describir los aspectos relacionados al proceso de enseñanza y aprendizaje nos situamos bajo la perspectiva socioconstructivista (Coll \& Colbs., 2002). Reconocemos la importancia de los principios que este enfoque psicológico plantea. La característica 
global que define este paradigma está relacionado con la construcción del conocimiento, originado desde la misma persona que aprende, pero no como un aprendiz inconexo, sino como un ser que interactúa con otros sujetos y que a la vez se sirve de experiencias, incorporándolas a su estructura cognitiva, para desarrollar sus conocimientos. La concepción socioconstructivista plantea ciertos principios básicos por los que se explica el funcionamiento del cerebro y con esto la adquisición del conocimiento. Un primer principio es el denominado: principio de auto-organización del sistema, proceso por el cual se forma la estructura cognitiva a través de una dinámica interna auto-producida (Büeler 1994 en Walber, 2005). Por medio de este proceso se determina que el aprendizaje se presenta cuando se forman estructuras de interacción, es decir, que el aprendiz no internaliza solamente la información extraída del contexto, sino que su sistema interno la modifica para propiciar una coexistencia aceptable entre éste y su medio ambiente conformando estas estructuras cognitivas (Maturana \& Varela, 2003). Esta interacción del sistema interno y el medio externo, llamada aprendizaje, formará el conocimiento. Puesto que el aprendizaje es una modificación entre el sistema interno de cada sujeto que aprende y el contexto, se podría afirmar que el aprendizaje no es posible moldearlo totalmente desde el exterior. Otro principio utilizado en la concepción socioconstructivista es el principio de autocontrol, en este principio se enmarca la reflexión, la toma de decisiones. En este sentido se podría mencionar que el aprendiz puede tener un control sobre los elementos de su aprendizaje como los objetivos de aprendizaje, los contenidos, el tiempo, los métodos y lugares de aprendizaje, siempre y cuando estemos hablando de un ambiente natural de aprendizaje, ya que los contextos escolares no se presentan como ambientes naturales de aprendizaje en vista de que los estudiantes deben aprender gran cantidad de contenidos para cumplir con los objetivos propuestos por el sistema educativo, por lo tanto el alumnado tiene la posibilidad de controlar ciertos elementos de su aprendizaje pero no todos (Pintrich, 2000). Un tercer principio se relaciona a la interacción de la enseñanza con el aprendizaje. En la teoría socioconstructivista se señala que en la enseñanza también se encuentra la función de auto-organización y auto-control como las funciones descritas anteriormente presentes en el proceso de aprendizaje. Esta función se puede evidenciar en los procesos que debe realizar el docente para organizar su clase, por ejemplo, en la elección de los objetivos, los contenidos, los métodos, etc. que utilizará con su alumnado. Previa a estas decisiones, el docente tuvo que haber observado el contexto de enseñanza, el nivel de conocimiento de sus estudiantes, los patrones de comportamientos del grupo, los recursos, etc. En este proceso previo de observación, el sistema interno del docente adquirió, asimiló y modificó la información previa con la nueva información que ya poseía sobre su grupo de estudiantes para decidir con qué elementos deberá contar para realizar su próxima actuación de enseñanza. Esta práctica permite una interacción entre el proceso de enseñanza y el proceso de aprendizaje, ya que la enseñanza se acoplará a las formas de aprender del grupo de estudiantes y viceversa (De la Fuente \& Justicia, 2007; Walber, 2005). Werlen y Bergamin (2012), mencionan la importancia de desarrollar competencias y motivaciones hacia una regulación del aprendizaje, tanto en aquellas personas que continúan una formación permanente no formal después de la educación obligatoria, como las que no continúan en dicha formación.

El desarrollo de las capacidades de autorregulación permiten al alumnado mantenerse motivados o evitar las distracciones al momento de aprender (Gaeta y Herrero, 2009). Así lo demuestran los diversos modelos teóricos del aprendizaje autorregulado como el adaptativo de Boekaerst (Boekaerts \& Niemivirta, 2000), el modelo metacognitivo orientado al proceso de Borkowski (Borkowski, Chan, \& Muthukrishna, 2000), el modelo de cuatro fases de Winne (Winne \& Perry, 2000), el modelo cognitivo y social de Zimmerman (Zimmerman, 2000) o el marco general de aprendizaje autorregulado de Pintrich (Pintrich, 2000). 
Paralelamente a estos cambios educativos, la incorporación de las nuevas tecnologías de la información y la comunicación (TIC) como recursos pedagógicos en las escuelas se ha incrementado notablemente (González Pérez \& De Pablos Pons, 2015). En este sentido, pretendemos analizar la contribución de estos nuevos instrumentos en el desarrollo del proceso de aprendizaje y de enseñanza (Decreto 72/2003, de 18 de marzo, de Medidas de Impulso de la Sociedad del Conocimiento en Andalucía).

Considerando la reciente y masiva implantación de estos medios en los centros educativos, como por ejemplo los "Centros TIC" en Andalucía (España), podemos encontrar un gran apoyo en las tecnologías de la información y comunicación. A través de ellos podemos lograr un enriquecedor trabajo de tipo colaborativo y participativo, favoreciendo el aprendizaje autónomo que será necesario a lo largo de la vida (Marco, 2008).

El estudio de Bernacki y otros (2010 en Werlen \& Bergamin, 2012), plantea la necesidad de desarrollar las competencias de autorregulación del aprendizaje ya que cada vez más los estudios no formales están utilizando contextos de aprendizaje virtuales como el "E-learning", "blended learning" o "mobile learning", donde una autoformación en manejo de las tecnologías y una correcta autorregulación del aprendizaje juegan un papel primordial para alcanzar exitosamente los objetivos de aprendizaje.

\section{Objetivos}

Los objetivos de nuestra investigación pretenden:

- Determinar qué tipo de estrategias metacognitivas de autorregulación utiliza el alumnado con mayor y menor frecuencia antes, durante la realización y al finalizar una actividad académica mediada por las tecnologías.

- Conocer la percepción del profesorado respecto a la utilización de estrategias metacognitivas de autorregulación en las tareas escolares de su alumnado antes, durante el proceso de realización y cuando finaliza la tarea.

- Establecer relaciones entre las percepciones del alumnado sobre su propio proceso de aprendizaje y la percepción del docente sobre el proceso de aprendizaje del alumnado.

\section{Metodología}

El diseño de nuestra investigación, atendiendo a los objetivos planteados, obedece a una investigación no experimental ya que no se manipula ninguna variable y es de tipo descriptiva explicativa, pues describe una característica o fenómeno de la realidad educativa (Buendía, Colás, \& Hernández, 1998). Optamos por el estudio transversal, pues las variables dependientes se miden solo una vez sobre diversos grupos preconfigurados que se comparan entre sí (Vallejo, Fernández-Cano y Torralbo, 2006) desde un enfoque cuantitativo, empleando estadísticos descriptivos en la evaluación de las estrategias metacognitivas de autorregulación.

\section{Diseño}

Basándonos en el modelo de autorregulación del aprendizaje de Zimmerman (2000), hemos establecido tres momentos en los que se ejecuta una tarea, los que hemos definido como Etapa antes, Etapa durante y Etapa final. 
Luego, basándonos en el modelo de autorregulación del aprendizaje propuesto por Pintrich (2000), hemos definido para cada fase y áreas señaladas por el autor, estrategias de aprendizaje que deberían estar presente en el aprendizaje autorregulado (Tabla 1).

\begin{tabular}{|c|c|c|c|}
\hline Etapa & Fases & Áreas & Estrategias \\
\hline \multirow[t]{4}{*}{ Antes } & \multirow[t]{4}{*}{$\begin{array}{l}\text { Previsión, } \\
\text { planificación y } \\
\text { activación }\end{array}$} & Cognición & $\begin{array}{l}\text { Establecimiento de metas a alcanzar } \\
\text { Activación del conocimiento previo y conocimientos } \\
\text { metacognitivos }\end{array}$ \\
\hline & & Motivación/afecto & $\begin{array}{l}\text { Creencias motivacionales: autoeficacia, metas, valor } \\
\text { asignado, interés personal. }\end{array}$ \\
\hline & & Conductual & Planificación del tiempo y esfuerzo \\
\hline & & Contextual & Percepción de la tarea y contexto \\
\hline \multirow[t]{8}{*}{ Durante } & \multirow[t]{4}{*}{ Supervisión } & Cognición & Toma de consciencia metacognitiva \\
\hline & & Motivación & Ser consciente de su patrón motivacional \\
\hline & & Conductual & Ser consciente de su propio comportamiento \\
\hline & & Contextual & $\begin{array}{l}\text { Ser consciente de las características de la tarea y del } \\
\text { contexto del aula }\end{array}$ \\
\hline & \multirow[t]{4}{*}{ Control } & Cognición & Uso de estrategias cognitivas \\
\hline & & Motivación & Estrategias motivacionales y de control emocional \\
\hline & & Conductual & Regulación del tiempo y del esfuerzo \\
\hline & & Contextual & Control de las tareas a realizar, clima y estructura de clase \\
\hline \multirow[t]{4}{*}{ Final } & \multirow[t]{4}{*}{$\begin{array}{l}\text { Reacción y } \\
\text { reflexión }\end{array}$} & Cognición & $\begin{array}{l}\text { Atribuciones que se realizan sobre las causas de sus } \\
\text { éxitos o fracasos }\end{array}$ \\
\hline & & Motivación & $\begin{array}{l}\text { Reacciones afectivas ante sus resultados como } \\
\text { consecuencia de las atribuciones }\end{array}$ \\
\hline & & Conductual & $\begin{array}{l}\text { Elección de la conducta que llevará a cabo en próximas } \\
\text { tareas }\end{array}$ \\
\hline & & Contextual & $\begin{array}{l}\text { Evaluaciones generales sobre la tarea y el ambiente de } \\
\text { clase }\end{array}$ \\
\hline
\end{tabular}

Una vez definidas las estrategias, se definieron conductas o técnicas más específicas que debería realizar el alumnado al momento de ejecutar una tarea de manera autorregulada, las que hemos utilizado en el Autoinforme que tuvo que responder el alumnado y los Registros de observación que tuvo que responder el profesorado (Tabla 2).

Tabla 2. Conductas o Técnicas que se observan en un alumno que autorregula su aprendizaje

Conductas o Técnicas

Escribo los pasos que voy a seguir para hacer la tarea

También escribo lo que pretendo hacer en cada paso

Me planteo realizar las tareas para aprender y saber cada vez más

Me propongo hacerlas cada vez mejor para obtener mejores calificaciones

Antes de iniciar la tarea, pienso en los conocimientos que tengo para realizarla con éxito

Antes de iniciar la tarea, pienso si tengo habilidades y experiencia para terminarla sin ayuda

Pensar en todas las cosas que tengo que hacer y sitios donde debo actuar, me da más seguridad para realizarla mejor

Cuando comienzo a realizar cada tarea, creo que puedo terminarla con éxito

Considero que las tareas son importantes y por ello, me gusta realizarlas independientemente de la calificación que obtenga

Me gusta sentir que el control de la realización de la tarea depende de mí mismo

Antes de iniciar su realización, observo las ventajas que me ofrecen las indicaciones y orientaciones del profesorado Antes de iniciar cada tarea, me aseguro de conocer bien las normas y plazos para realizarla y entregarla

Sigo el plan acordado para desarrollar la tarea en todos sus pasos 
Estoy atento y anoto los cambios que surgen en la realización, sobre el plan inicial de trabajo y plazos de entrega de la tarea

Decido, sin ayuda, el tipo de acciones que debo emplear en cada paso de la realización de la tarea

Soy consciente de mi capacidad para terminar con éxito las tareas que me encomiendan

Realizo las tareas lo mejor posible, porque me importan las recompensas o calificaciones que obtenga por ello

Controlo, si lo que voy haciendo está bien, porque considero importantes los aprendizajes y habilidades que adquiero

Suelo controlar mis emociones y sentimientos, que se producen por conflictos o dificultades no previstos

Cuando surgen dificultades, soy consciente y controlo el esfuerzo y tiempo que he de aumentar para finalizarla

Cuando lo considero conveniente, modifico la planificación inicial de la tarea, proponiendo otras formas y materiales no previstos

Propongo diferentes formas de trabajo individual o en grupo para que el ambiente de trabajo favorezca la terminación con éxito de las tareas

Entrego mis tareas en el tiempo acordado

Yo valoro todo el proceso y resultado de la tarea, y lo anoto para tenerlo en cuenta en otras ocasiones

Yo valoro lo que he aprendido con la tarea, lo anoto y digo

Pienso en las dificultades que he tenido durante la realización. Las anoto o digo

Valoro mi satisfacción o no satisfacción por realizar las tareas en las condiciones acordadas con el profesorado

Valoro la capacidad que he adquirido para realizar tareas más o menos iguales

Pienso y apunto si el ambiente de clase ha facilitado o dificultado la realización de la tarea

Se ha utilizado un solo diseño tanto para el Autoinforme como para el Registro de observación, solo varía la formulación de la pregunta ya que para el Autoinforme los ítems están redactados en 1ra. persona y los ítems del Registro de observación se encuentran redactados en 3ra. persona.

\section{Participantes}

Para seleccionar los centros educativos participantes en la investigación, de acuerdo a los criterios requeridos para el estudio, se realizó un muestreo no probabilístico por conveniencia. La muestra estuvo compuesta por 7 centros de Educación Infantil y Primaria de Granada capital. Solo un centro es concertado y los seis restantes son públicos.

Estos centros públicos participan en el Programa "Plan Escuela TIC 2.0" de la Junta de Andalucía, donde cada alumno de 5to y 6to nivel de primaria ha recibido un ordenador portátil para llevar a cabo sus actividades escolares diarias, la dotación de ordenadores para los profesores tutores de estos niveles, la implementación de pizarras digitales interactivas (PDI) y proyectores multimedia para cada aula de estos niveles educativos, por lo que el conjunto de estos medios digitales y su utilización diaria y en cada asignatura, permiten que el contexto escolar pase de ser un contexto escolar convencional a un contexto escolar "digital" o "TIC" ( (Decreto 72/2003, de 18 de marzo, de Medidas de Impulso de la Sociedad del Conocimiento en Andalucía; Cubillo, 2010).

La muestra de alumnado está constituida por 332 estudiantes de $5^{\circ}$ y $6^{\circ}$ curso de Educación Primaria donde las edades oscilan entre los 10 y 12 años. El $47.3 \%$ son mujeres (157 alumnas). El 52.7\% de la muestra son chicos (175 alumnos) (Tabla 3 ). 


\begin{tabular}{ccc}
\hline \multicolumn{3}{c}{ Tabla 3. Descriptivos de la muestra por nivel y género alumnos } \\
\hline \multicolumn{3}{c}{ Descriptivos } \\
\hline Nivel & $N=332$ \\
$5^{\circ}$. & 115 & $34.6 \%$ \\
$6^{\circ}$. & 217 & $65.4 \%$ \\
Género & $N=332$ & \\
Femenino & 157 & $47.3 \%$ \\
Masculino & 175 & $52.7 \%$ \\
\hline
\end{tabular}

La muestra de tutores se constituye por 18 profesores-tutores, de los cuales 11 fueron hombres y 7 fueron mujeres (Tabla 4).

\begin{tabular}{ccc}
\hline Tabla 4. Descriptivos de la muestra por nivel y género profesores-tutores \\
\hline \multicolumn{3}{c}{ Descriptivos } \\
\hline Nivel & $\mathrm{N}=18$ \\
5to. & 9 & $50.0 \%$ \\
6to. & 9 & $50.0 \%$ \\
Género & $\mathrm{N}=18$ & \\
Femenino & 7 & $38.9 \%$ \\
Masculino & 11 & $61.1 \%$ \\
\hline
\end{tabular}

\section{Variables}

Las variables de estudio que se van a medir son las siguientes: alumno; profesor-tutor; género; centro y nivel. Las variables para la etapa antes:

- Estrategias de autorregulación de planificación: Referida a las acciones de planificación cognitiva (establecimiento de metas a alcanzar; activación del conocimiento previo y conocimientos metacognitivos), emocionales (activación de las creencias motivacionales como: la autoeficacia, metas, valor asignado, interés personal; emociones), conductuales (planificación del tiempo y esfuerzo) y contextuales (percepción de la tarea y contexto) a las que recurre el alumno antes de comenzar la tarea. Estas actividades permiten al alumno tener una visualización de los recursos con los que cuenta, ya sean de carácter interno o externo y proyectar su actuar durante el desarrollo de la actividad presentada (Pintrich, 2000).

Las variables para la etapa durante:

- Estrategias de autorregulación de auto-observación: Se consideran las acciones de supervisión cognitivas (Toma de consciencia metacognitiva) emocionales (ser consciente del patrón motivacional), conductuales (ser consciente de su propio comportamiento) y contextuales (ser consciente de las características de la tarea y del contexto del aula). Estas estrategias introspectivas le permiten al alumno verificar si su actuar se está ajustando a los requerimientos de la tarea (Pintrich, 2000).

- Estrategias de control: Las acciones de control cognitivo (uso de estrategias cognitivas), emocionales (estrategias motivacionales y de control emocional), conductuales (regulación del tiempo y del esfuerzo) y contextuales (control de las tareas a realizar, clima y estructura de clase) le permiten al alumno ajustar su accionar para mantener la dirección correcta durante la realización de la tarea (Pintrich, 2000). 
Las variables para la etapa final:

- Estrategias de evaluación: Las acciones de evaluación cognitivas (atribuciones que se realizan sobre las causas de sus éxitos o fracasos), emocionales (reacciones afectivas ante sus resultados como consecuencia de las atribuciones), conductuales (elección de la conducta que llevará a cabo en próximas tareas) y contextuales (evaluaciones generales sobre la tarea y el ambiente de clase) permiten al alumno reflexionar y valorar los resultados obtenidos y mantenerlos o adecuarlos, según sea la pertinencia, en las tareas futuras (Pintrich, 2000).

\section{Instrumentos}

Los instrumentos utilizados han sido el Autoinforme sobre procesos de aprendizaje mediados por TIC (dirigido al alumnado) y Registro observacional sobre procesos de aprendizaje mediados por TIC (dirigido al profesorado), diseñados para conocer la mejora de los procesos que utiliza el alumnado para aprender cuando lo hace a través de Tecnologías de información y comunicación.

\section{a) Autoinforme sobre procesos de aprendizaje mediados por TIC}

Este Autoinforme tiene por objetivo conocer lo que piensa y hace el alumnado antes de iniciar las tareas que se encomiendan en clase, durante el proceso de su realización y cuando la finaliza. El autoinforme consta de 29 ítems distribuidos en tres dimensiones que responden a tres preguntas: i) ¿qué sueles hacer antes de comenzar cada tarea escolar que te demanda tu profesor/a? (compuesto por 12 ítems); ii) ¿qué sueles hacer durante la realización de la tarea? (compuesto por 11 ítems; iii) ¿qué sueles hacer una vez que finalizas la tarea? (compuesto por 6 ítems). Las opciones de respuesta comprendían tres alternativas, en este caso, el alumnado debía contestar a un grupo de acciones, señalando la columna que más se acercaba a lo que realmente hace: siempre, pocas veces o nunca.

El Autoinforme se aplicó, por el investigador, simultáneamente a cada alumno del grupo, en una sola sesión de treinta minutos aproximadamente previa coordinación con el profesor tutor de cada grupo de alumnos.

Para estudiar la fiabilidad del autoinforme, se calculó la consistencia interna, de cada dimensión del instrumento, a través de coeficiente Alfa de Cronbach, utilizando el programa SPSS Statistics 21, obteniendo los siguientes resultados (Tabla 5):

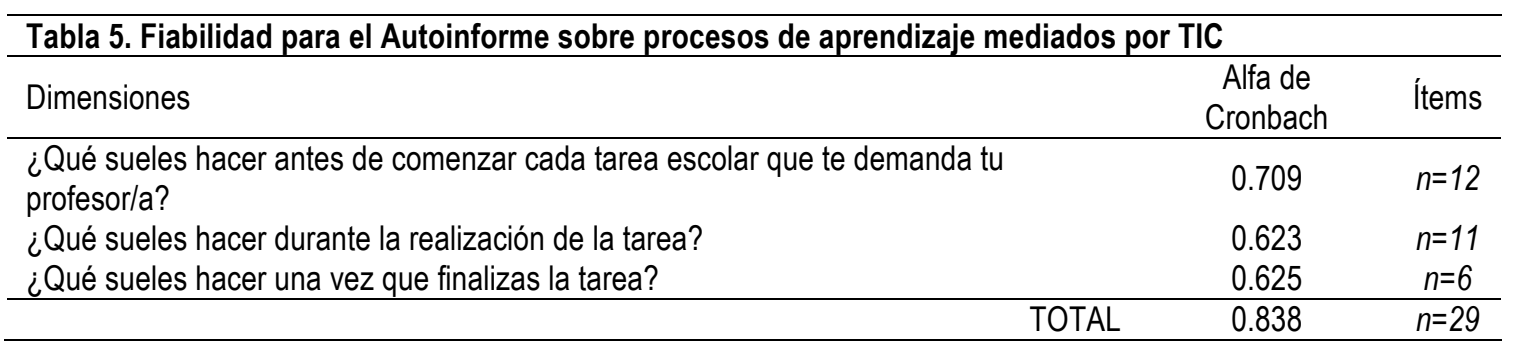

Como se muestra en la Figura 1, la validación de la estructura del autoinforme dirigido al alumnado, con la muestra que conforma este estudio, se ha realizado mediante modelos de ecuaciones estructurales. La evaluación de bondad del modelo indica un buen ajuste global a los datos empíricos en todos los índices. Así, Chi-cuadrado presenta un valor asociado de $\rho$ no significativo $\left(\chi^{2}=65.80 ; g l .=48 ; \rho=.054\right)$. El valor del 
índice comparativo de ajuste $(\mathrm{CFI})$ respecto a un modelo nulo presenta un valor (.91) superior al recomendado. El valor de AGFI (.84) muy cercano al valor recomendado. El valor de RMSEA (.043) es inferior al valor recomendado. En suma, todos los índices presentados indican la existencia de un buen ajuste del modelo a los datos empíricos, respecto a las relaciones significativas entre los constructos. Para que exista un buen ajuste, los valores GFI, CFI y AGFI deberían ser cercanos al valor de 0,85 (cuanto mayor sea el valor, mejor ajuste), y el valor RMSEA debería ser cercano a 0,05 (cuanto menor sea el valor, mejor ajuste) (Byrne, 2001).

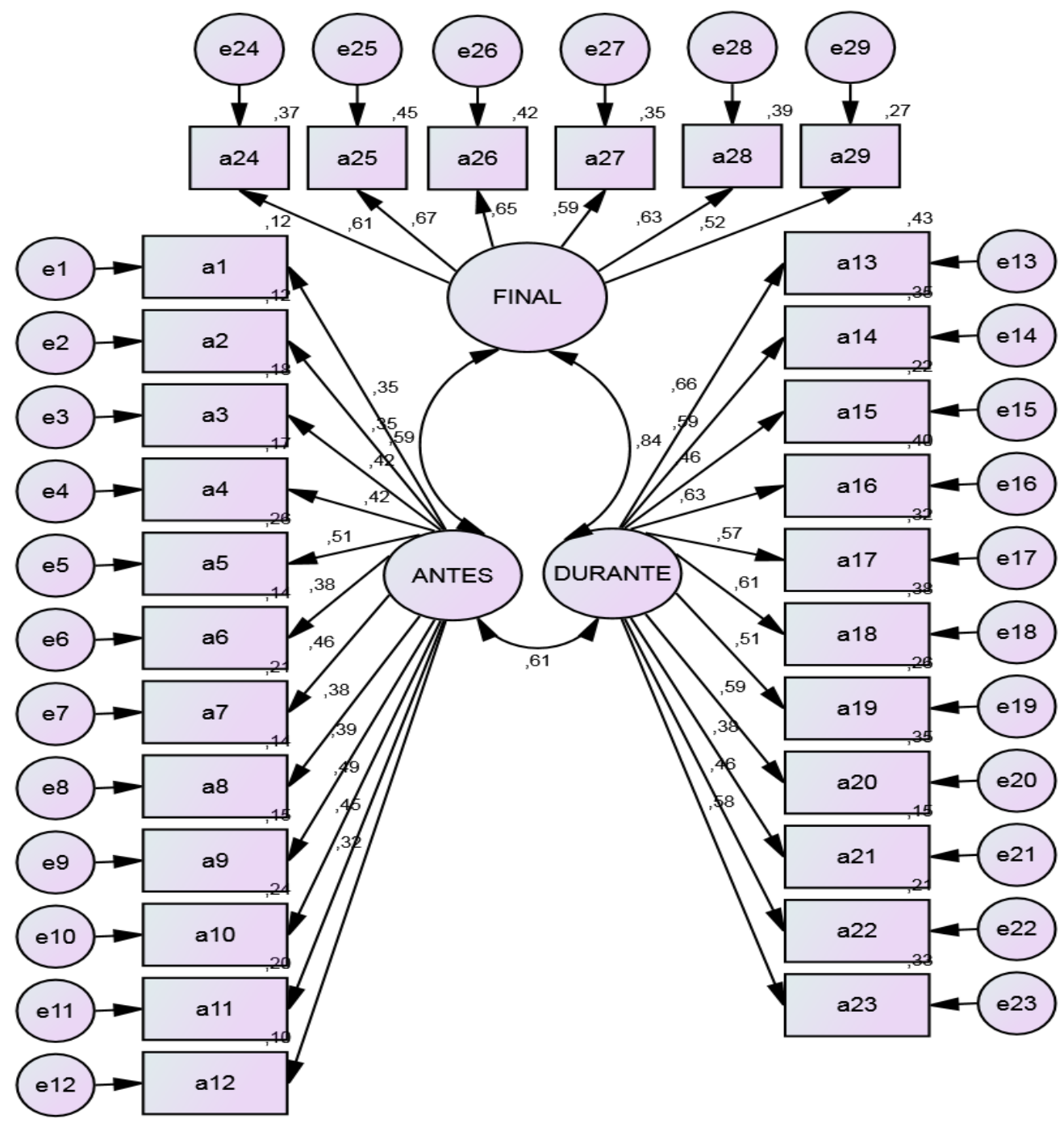

Figura 1. Modelo de ecuaciones estructurales del Autoinforme

\section{b) Registro observacional sobre procesos de aprendizaje mediados por TIC}

Este Registro observacional tiene por objetivo conocer los procesos que utiliza el alumnado para aprender en un aula mediada por las TIC, desde la percepción del profesorado. Para ello, el profesor-tutor debería registrar determinados comportamientos del alumnado cuando está realizando sus tareas escolares: antes, durante el proceso de su realización y cuando la finaliza. El registro observacional consta de 29 ítems distribuidos en tres dimensiones que responden a tres preguntas: i) ¿qué suele hacer el alumno/a antes de comenzar cada tarea escolar? (compuesto por 
12 ítems); ii) ¿qué suele hacer durante la realización de la tarea? (compuesto por 11 ítems; iii) ¿qué suele hacer una vez que finaliza la tarea? (compuesto por 6 ítems). Las opciones de respuesta comprendían tres alternativas, en este caso, el profesorado debía contestar a un grupo de acciones, señalando la columna que más se acercaba a lo que realmente ha observado en sus alumnos: siempre, pocas veces o nunca.

Los registro de observación fueron entregados a los profesores tutores para su cumplimentación, la que se llevó a cabo en un plazo de entre tres a cuatro semanas. Después de este plazo fueron pasados a recoger por el investigador a cada centro educativo.

Para estudiar la fiabilidad del Registro de observación, se calculó la consistencia interna, de cada dimensión del instrumento, a través de coeficiente Alfa de Cronbach, obteniendo una alta consistencia (.971) (Tabla 6):

\begin{tabular}{|c|c|c|}
\hline Dimensiones & Alfa de Cronbach & Items \\
\hline $\begin{array}{l}\text { ¿Qué suele hacer antes de comenzar cada tarea escolar que le } \\
\text { demanda el/la profesor/a? }\end{array}$ & 0.938 & $n=12$ \\
\hline $\begin{array}{l}\text { ¿Qué suele hacer durante la realización de la tarea? } \\
\text { ¿Qué suele hacer una vez que finaliza la tarea? }\end{array}$ & $\begin{array}{l}0.936 \\
0.897\end{array}$ & $\begin{array}{l}n=11 \\
n=6\end{array}$ \\
\hline 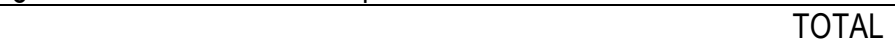 & 0.971 & $n=29$ \\
\hline
\end{tabular}

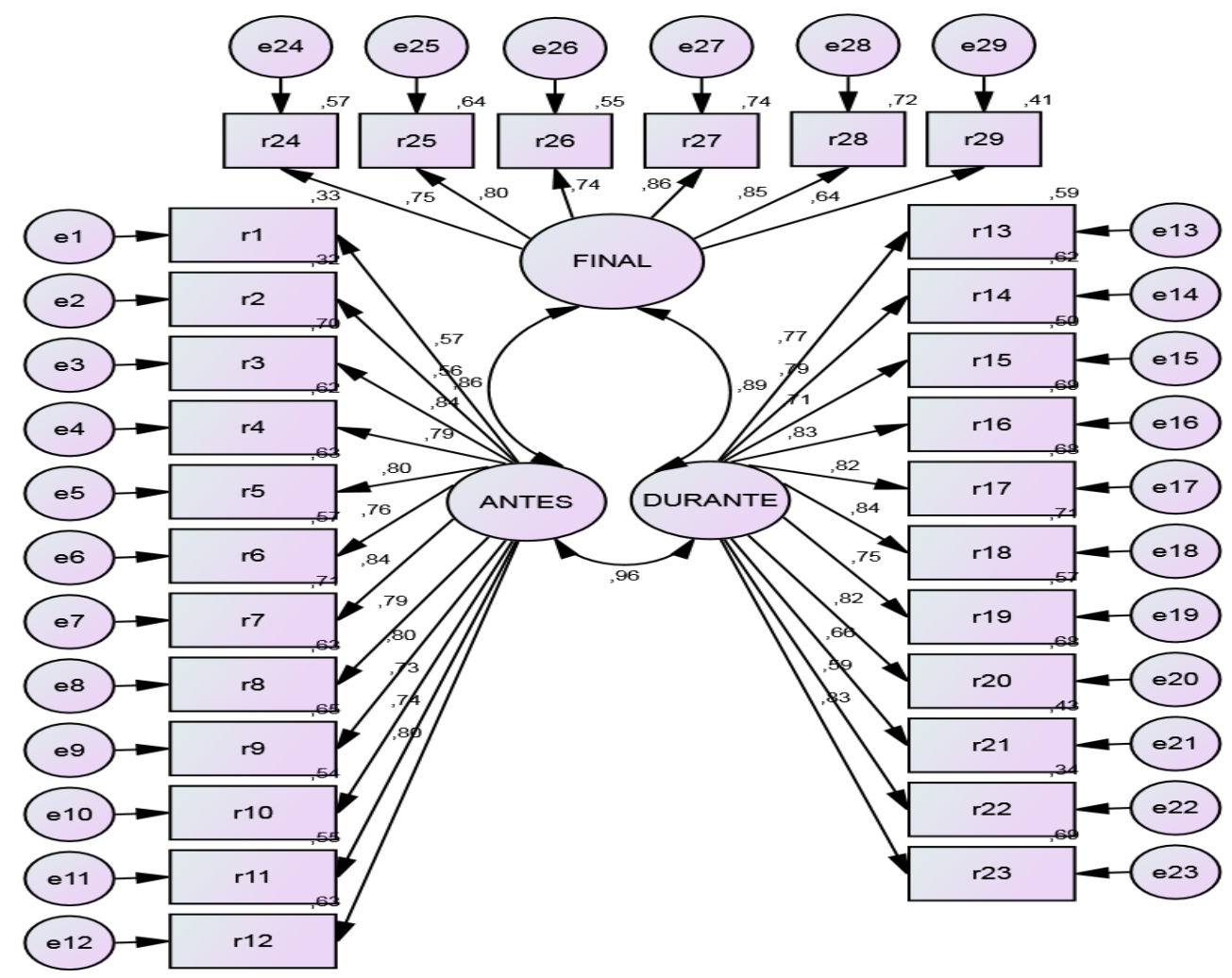

Figura 2. Modelo de ecuaciones estructurales del Registro observacional

Como se muestra en la Figura 2, la validación de la estructura del Registro observacional dirigido al profesorado, con la muestra que conforma este estudio, se ha realizado mediante modelos de ecuaciones estructurales. La evaluación de bondad del 
modelo indica un buen ajuste global a los datos empíricos en todos los índices. Así, Chi-cuadrado presenta un valor asociado de $\rho$ no significativo $\left(\chi^{2}=985.90 ; \mathrm{gl} .=274\right.$; $\rho=.005)$. el valor del índice comparativo de ajuste $(C F I)$ respecto a un modelo nulo presenta una valor (.89) superior al recomendado. El valor de AGFI (.84) es muy cercano al valor recomendado. El valor de RMSEA (.08) es cercano al valor recomendado. En suma, todos los índices presentados indican la existencia de un buen ajuste del modelo a los datos empíricos, respecto a las relaciones significativas entre los constructos.

\section{Análisis de los datos y Resultados}

\section{Resultados del Autoinforme}

Podemos observar, para la etapa antes, que los recursos metacognitivos más utilizados por el alumnado se encuentran relacionados con: Proponerse metas de mejora personal (87.3\%), Aumentar su seguridad para el desarrollo de la tarea al pensar en todas las cosas que tiene que hacer y sitios donde debe actuar $(77.4 \%)$ y Sentir que el control de la realización de la tarea depende de sí mismo (76.5\%). Los recursos metacognitivos que el alumnado declara utilizar con menor frecuencia para esta etapa se encuentran relacionados con: Pensar en lo que va a hacer en cada paso de la tarea (38.6\%), pensar en cada paso que va a seguir para hacer la tarea $(35.5 \%)$ y pensar sobre sus habilidades y experiencias previas para realizar la tarea sin ayuda $(13.0 \%)$.

Para la etapa durante, los recursos metacognitivos más utilizados por el alumnado se encuentra relacionado a estrategias como: tratar de controlar su motivación intrínseca con recompensas extrínsecas (81.3\%), supervisar si está siguiendo correctamente las indicaciones de la tarea (76.2\%) y tratar de controlar su autoeficacia (76.2\%). Los recursos metacognitivos a los que menos recurren los estudiantes durante esta etapa se encuentran relacionados con: proponer diferentes formas de trabajo, individual o en grupo, para que el ambiente de trabajo favorezca la terminación con éxito de las tareas (20.2\%), modificar la planificación inicial de la tarea en caso necesario para asegurar el éxito de la tarea (19.9\%) y controlar sus emociones negativas (10.2\%).

En la etapa final, los recursos metacognitivos más utilizados por el alumnado se encuentra relacionado con: la valoración de la satisfacción de la tarea realizada (69.6\%), valoración de las capacidades adquiridas para ser utilizadas en próximas actuaciones (65.4\%) y valoración de los conocimientos adquiridos (del contenido) (39.2\%). Para esta etapa los recursos metacognitivos menos utilizados son: analizar si el ambiente de clases ha favorecido a la realización de la tarea $(24.1 \%)$, valoración de todo el proceso y resultado de la tarea (19.3\%) y pensar en las dificultades que se le han presentado en la tarea $(17.8 \%)$.

\section{Resultados del registro de observación}

Con respecto al registro observacional, cuyo objetivo era conocer la percepción del profesorado respecto a la utilización de estrategias metacognitivas de autorregulación en las tareas escolares, podemos observar para la etapa antes, que los recursos metacognitivos más utilizados por sus estudiantes son: proponerse metas de mejora personal (73.5\%), planificación del tiempo y del esfuerzo para la realización de la tarea $(65.7 \%)$ y activación de los juicios de auto-eficacia (63.3\%). Según la percepción de los profesores los recursos metacognitivos que menos utilizan los estudiantes para esta etapa son: Pensar en lo que va a hacer en cada paso que va a seguir para hacer la tarea $(24.1 \%)$, pensar en cada paso que va a seguir para hacer la tarea $(18.4 \%)$ y 
pensar sobre sus habilidades y experiencias previas para realizar la tarea sin ayuda $(12.3 \%)$.

Para la etapa durante, la percepción del profesorado sobre la utilización de los recursos metacognitivos más utilizados por su alumnado son: tratar de controlar su motivación intrínseca con recompensas extrínsecas (66.9\%), supervisar si está siguiendo correctamente las indicaciones de la tarea $(66.6 \%)$ y tratar de influir sobre las indicaciones de la tarea $(65.4 \%)$. Los recursos metacognitivos que los profesores consideran que son los menos utilizados por el alumnado durante esta etapa se relacionan con: proponer diferentes formas de trabajo, individual o en grupo, para que el ambiente de trabajo favorezca la terminación con éxito de las tareas (30.7\%), modificar la planificación inicial de la tarea en caso necesario para asegurar el éxito de ésta $(26.5 \%)$ y supervisar y controlar el tiempo y el esfuerzo para alcanzar a terminar la tarea en el plazo establecido (13.0\%).

Para la etapa final, podemos observar que los recursos metacognitivos que más utiliza el alumnado, según la percepción del profesorado, se encuentran relacionados con: la valoración de la satisfacción de la tarea realizada (59.0\%), valoración de las capacidades adquiridas para ser utilizadas en próximas actuaciones $(55.7 \%)$ y valoración de los conocimientos adquiridos (del contenido) (51.2\%). Y los recursos metacognitivos menos utilizados en esta etapa son: analizar si el ambiente de clases ha favorecido a la realización de la tarea $(28.9 \%)$, valoración de todo el proceso y resultado de la tarea (16.6\%) y pensar en las dificultades que se le han presentado en la tarea $(14.8 \%)$.

\section{Resultados del análisis de asociación del Autoinforme y el Registro observacional}

Para comprobar si existe o no coincidencia entre ambas opiniones hemos realizado un análisis de correlaciones. Previo a la realización de dichos análisis hemos aplicado las pruebas de bondad de ajuste y verificación de supuestos Kolmogorov - Smirnov, para comprobar las condiciones de normalidad de la muestra.

Según los resultados obtenidos en la prueba de Kolmogorov-Smirnov para una muestra, la significación de las dimensiones medida al 0.05 es de $(0<0.05)$, por lo que rechazamos la hipótesis nula, ya que los datos proceden de una distribución anormal.

De acuerdo a estos resultados aplicaremos la prueba de correlación de Spearman para establecer correlaciones entre las distintas variables que conforman la opinión que tiene el alumnado sobre las concepciones de su proceso de aprendizaje y las percepciones que tiene el profesorado sobre el proceso de aprendizaje de su alumnado.

Hemos definido una hipótesis nula y una hipótesis confirmatoria utilizada para cada una de las estrategias por igual:

- $\mathrm{H}_{0}$ : No existe diferencia entre la opinión del alumno frente a la opinión del profesor.

- $\mathrm{H}_{1}$ : Existe diferencia entre la opinión del alumno frente a la opinión del profesor.

En la Tabla 7 se observa que no existe diferencia entre la opinión de los estudiantes frente a la de los profesores-tutores en la mayoría de las estrategias excepto en la estrategia 10 y 12. 


\begin{tabular}{|c|c|c|c|}
\hline \multicolumn{4}{|l|}{$\mathrm{N}=332$} \\
\hline $\begin{array}{l}\text { Estrategia } \\
\mathrm{N}^{0}\end{array}$ & Estrategia de autorregulación & $\begin{array}{l}\text { Coeficiente de } \\
\text { correlación de } \\
\text { Spearman }\end{array}$ & $\begin{array}{c}\text { Sig. } \\
\text { (bilateral) }\end{array}$ \\
\hline 1 & $\begin{array}{l}\text { Pensar en los pasos que el alumno debe seguir para realizar } \\
\text { la tarea }\end{array}$ & -.077 & .160 \\
\hline 2 & $\begin{array}{l}\text { Pensar en lo que va a hacer el alumno en cada paso de la } \\
\text { tarea }\end{array}$ & -.025 & .652 \\
\hline 3 & Proponerse metas de aprendizaje (aumento del conocimiento) & 104 & .059 \\
\hline 4 & $\begin{array}{l}\text { Proponerse metas de mejora personal (mejora de sí mismo, } \\
\text { de sus habilidades u otros) }\end{array}$ & .059 & .288 \\
\hline 5 & $\begin{array}{l}\text { Tratar de recordar si tiene información previa sobre el } \\
\text { contenido de la tarea }\end{array}$ & .060 & .275 \\
\hline 6 & Pensar si se encuentra preparado para desarrollar la tarea & .013 & .819 \\
\hline 7 & $\begin{array}{l}\text { Aumentar su seguridad para el desarrollo de la tarea al } \\
\text { pensar en todas las cosas que tiene que hacer y sitios donde } \\
\text { debe actuar }\end{array}$ & -.057 & .297 \\
\hline 8 & $\begin{array}{l}\text { Pensar sobre el nivel de seguridad en sí mismo que siente al } \\
\text { enfrentarse a la tarea }\end{array}$ & .045 & .410 \\
\hline 9 & Pensar en la importancia/valor de la tarea & .025 & 648 \\
\hline 10 & Prever el nivel de dificultad de la tarea & $.113\left(^{*}\right)$ & .040 \\
\hline 11 & Conocer las indicaciones de cómo deberá desarrollar la tarea & .018 & .743 \\
\hline 12 & $\begin{array}{l}\text { Conocer las normas establecidas para el desarrollo de la } \\
\text { tarea y de aula }\end{array}$ & $.155\left({ }^{* *}\right)$ & .005 \\
\hline
\end{tabular}

En la Tabla 8 se observa que existe diferencia entre la opinión del alumnado frente a la de los profesores en la mayoría de las estrategias excepto sobre las estrategias 15 , 19,20 y 21.

\begin{tabular}{|c|c|c|c|}
\hline \multicolumn{4}{|l|}{$\mathrm{N}=332$} \\
\hline $\begin{array}{c}\text { Estrategia } \\
\mathrm{N}^{0}\end{array}$ & Estrategia de autorregulación & $\begin{array}{c}\text { Coeficiente de } \\
\text { correlación de Spearman }\end{array}$ & $\begin{array}{c}\text { Sig. } \\
\text { (bilateral) }\end{array}$ \\
\hline 13 & $\begin{array}{l}\text { Estar consciente si está entendiendo o no las } \\
\text { indicaciones de cómo desarrollar la tarea. }\end{array}$ & $.126\left({ }^{*}\right)$ & .022 \\
\hline 14 & $\begin{array}{l}\text { Estar consciente sobre si se está adecuando o no a las } \\
\text { normas de aula }\end{array}$ & $.111\left(^{*}\right)$ & .044 \\
\hline 15 & $\begin{array}{l}\text { Decidir cuándo y qué tipo de estrategias cognitivas } \\
\text { debe utilizar durante el desarrollo de la tarea }\end{array}$ & .071 & .195 \\
\hline 16 & Tratar de controlar su auto-eficacia & $.122\left(^{*}\right)$ & .027 \\
\hline 17 & $\begin{array}{l}\text { Tratar de controlar su motivación extrínseca con } \\
\text { recompensas extrínsecas }\end{array}$ & $\left..146^{(* *}\right)$ & .008 \\
\hline 18 & $\begin{array}{l}\text { Tratar de controlar su motivación intrínseca } \\
\text { aumentando el valor de la tarea }\end{array}$ & $\left..168^{* *}\right)$ & .002 \\
\hline 19 & $\begin{array}{l}\text { Tratar de controlar sus afectos negativos como la } \\
\text { ansiedad }\end{array}$ & .099 & .073 \\
\hline 20 & $\begin{array}{l}\text { Adecuarse a los tiempos estimados para el desarrollo } \\
\text { de la tarea }\end{array}$ & .044 & .422 \\
\hline 21 & $\begin{array}{l}\text { Controlar su esfuerzo en relación a la dificultad de la } \\
\text { tarea }\end{array}$ & -.013 & .813 \\
\hline 22 & Tratar de influir sobre el ambiente del aula & $\left..1599^{* *}\right)$ & .004 \\
\hline 23 & Tratar de influir sobre las indicaciones de la tarea & $\left..341^{(* *}\right)$ & .000 \\
\hline
\end{tabular}


Las diferencias entre las opiniones del alumnado y del profesorado no son significativas según la prueba de Spearman para la dimensión final, como lo indican los valores en la Tabla 9.

\begin{tabular}{|c|c|c|c|}
\hline \multicolumn{4}{|l|}{$N=332$} \\
\hline Estrategia $\mathrm{N}^{0}$ & Estrategia de autorregulación & $\begin{array}{l}\text { Coeficiente de } \\
\text { correlación de } \\
\text { Spearman }\end{array}$ & Sig. (bilateral) \\
\hline 24 & Evaluar el resultado total de la tarea & -.020 & .711 \\
\hline 25 & $\begin{array}{l}\text { Evaluar si ha aumentado o no su conocimiento } \\
\text { sobre el contenido }\end{array}$ & .083 & .129 \\
\hline 26 & Evaluar la dificultad propia de la tarea & .064 & .244 \\
\hline 27 & $\begin{array}{l}\text { Evaluar su nivel de satisfacción /insatisfacción de su } \\
\text { desempeño }\end{array}$ & .045 & .417 \\
\hline 28 & $\begin{array}{l}\text { Reflexionar sobre los cambios en su desempeño } \\
\text { futuro }\end{array}$ & .075 & .174 \\
\hline 29 & $\begin{array}{l}\text { Reflexiona acerca de los aspectos negativos y } \\
\text { positivos del ambiente del aula }\end{array}$ & -.051 & .358 \\
\hline
\end{tabular}

Según los valores arrojados en la prueba de correlación de Spearman realizados a las cuatro dimensiones analizadas sobre el tipo de estrategia metacognitiva de: Planificación, auto-observación, control y evaluación podemos establecer que existen diferencias significativas entre la opinión del alumnado frente a la del profesorado en la dimensión auto-observación y control, lo podemos observar en la Tabla 10.

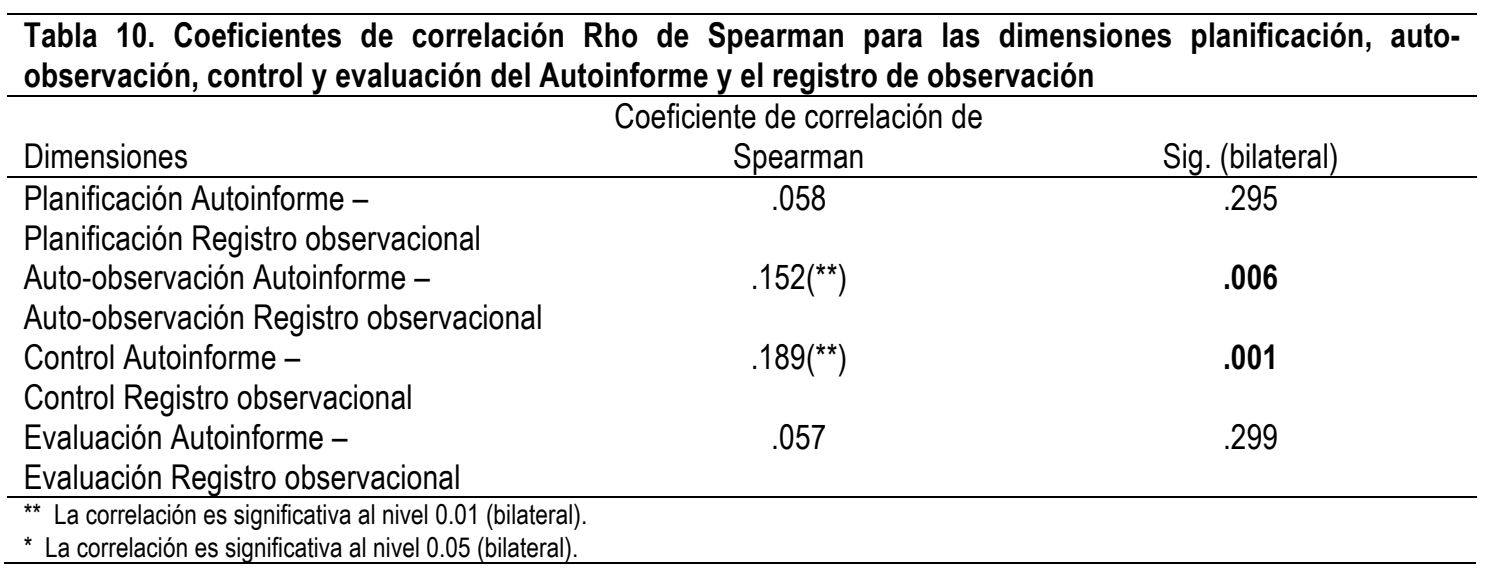

\section{Conclusiones}

De acuerdo a los resultados obtenidos podemos concluir que el alumnado reconoce utilizar estrategias de aprendizaje que consideramos bastante importante, con las cuales se sirven para facilitar, mejorar y lograr resultados académicos exitosos desde la propia autorregulación de su aprendizaje.

Describimos a continuación las tres primeras estrategias mayormente utilizadas por los estudiantes y cómo éstas influyen positivamente en el rendimiento académico. Para la etapa antes, las estrategias de planificación como proponerse metas de aprendizaje, con las que orientan la ejecución, la supervisión y la evaluación de la cognición, persiguiendo los objetivos de la tarea para alcanzar buenos resultados académicos y/o de mejora personal (Pintrich, 2000); también activan los juicios de 
auto-eficacia, estrategia de tipo motivacional, con la que ellos se sienten seguros de sus capacidades cognitivas, proyectándose a sí mismos capaces de realizar la tarea con éxito, permitiéndose tener una visión optimista de su desempeño académico, influyendo positivamente en su esfuerzo, voluntad, persistencia, rendimiento y el aprendizaje (Pintrich, 2000); y la estrategia de tipo conductual relacionada a la gestión del tiempo y del esfuerzo que les permite planificar con cuanto tiempo deberán contar para la realización de la actividad y en qué medida deberán esforzarse según ellos visualicen la dificultad de la tarea propuesta, previendo tener el control de la realización de ésta en todo momento.

Para la etapa durante, el alumnado reconoce utilizar en mayor porcentaje las estrategias de control como: las estrategias de tipo motivacional y de control emocional relacionadas al control de la auto-eficacia, con la que los estudiantes se motivan a sí mismos a continuar con la tarea de manera correcta, aumento de la motivación intrínseca recurriendo a recompensas extrínsecas y la estrategia de supervisión, en el área contextual, manteniéndose consciente de las características de la tarea y el contexto del aula considerando las oportunidades y limitaciones que están interviniendo en la realización de la actividad, en palabras de Pintrich (2000, p. 470) "...la conciencia y el control de las normas de aula, las prácticas de calificación, requisitos del trabajo, las estructuras de recompensa y el comportamiento de los docentes en general son muy importantes para que los estudiantes estén bien en el aula" por lo tanto mantener una supervisión y un control de estos elementos le permite al alumnado ajustarse a las necesidades de la tarea y el contexto para mantenerse en el camino correcto hacia la meta propuesta en la etapa anterior.

Para la etapa después, podemos observar que las estrategias utilizadas en mayor porcentaje son: estrategias de reacción y reflexión del área motivacional tal como la evaluación del desempeño con la que el alumnado realiza una valoración de los resultados, reflexionando sobre éstos y atribuyendo razones por las cuales se alcanzaron esos resultados y no otros "...estas reflexiones y reacciones pueden llevar a cambios en los niveles futuros de la auto-eficacia y la expectativa de éxito futuro, así como el valor y el interés" (Pintrich, 2000 p. 466). Seguida de esta estrategia se presentó, tal como se menciona en la cita anterior, una de tipo conductual como es la elección de la conducta que llevará a cabo en próximas tareas de acuerdo a la valoración realizada a los elementos que puso en ejecución para desarrollar la tarea, esta valoración de su conducta le permitirá al alumnado volver a poner en práctica las estrategias que ellos consideran adecuadas guiándose por su experiencia previa con las que alcanzó resultados exitosos descartando aquellas que no le sean tan propicias para el tipo de actividad a desarrollar.

Hemos observado que las estrategias de tipo contextual son a las que menos recurren, puede ser debido a que el alumnado tiene menor control sobre éstas, ya que los factores contextuales se encuentran relacionados, por ejemplo, al lugar físico, normas, situaciones, etc. con las que se encuentra el alumnado en el momento de desarrollar la tarea pero el "cómo se presentan" no dependen de ellos (Pintrich, 2000).

De acuerdo a los resultados del registro de observación aplicado al profesorado, observamos que no existe diferencia significativa en la mayoría de las estrategias para la etapa antes, por lo tanto podemos deducir que el profesorado tiende a conocer como el alumnado planifica y da los primeros pasos al enfrentarse a una tarea.

Para la etapa durante, se presentan más diferencias significativas entre las opiniones del alumnado y el profesorado, por lo que podemos suponer que en esta etapa son muchas más variadas las posibilidades de estrategias que se pueden utilizar en 
comparación a la etapa antes y final, también en esta etapa se recurre mucho más a procesos metacognitivos, en el proceso de supervisión y control las estrategias son más de tipo intrínsecas como el "estar consciente de..." o de "supervisión y control de la autoeficacia", las que son menos observables.

Por último, para la etapa final, no se presentaron diferencia significativas, es decir que el profesorado conoce muy bien como sus alumnos realizan el proceso de evaluación al finalizar la tarea, puede ser debido a que el número de estrategias de esta etapa son más limitadas o son más observables por ejemplo si las revisiones de las tareas se hacen de forma grupal con toda la clase.

Por lo tanto podemos concluir que en los contextos educativos donde se trabaja con TIC los alumnos recurren a estrategias de aprendizaje de manera recurrente, y que esta opinión del alumnado es corroborada con la percepción del profesorado al existir bastante coincidencia entre ambas opiniones. También se determina que son las estrategias de tipo motivacional las que más utiliza el alumnado, que hubo mayor coincidencia de opiniones de los estudiantes y el profesorado sobre el uso de estrategias de tipo cognitivas y que el alumnado declara utilizar las estrategias de control a diferencia del profesorado que perciben que los estudiantes recurren más a las estrategias de auto-observación.

Como nuestro estudio tenía como objetivo analizar la presencia de estrategias de aprendizaje que permiten la autorregulación del aprendizaje en el alumnado en contextos TIC, en los que todas las actividades académicas son desarrolladas a través de ordenadores, pantallas digitales u otros medios digitales, sería interesante conocer también si existen diferencias en la utilización de estas estrategias entre aulas de tipo convencional y aulas donde se apliquen las TIC y cómo influyen sobre el rendimiento académico, quizás podemos plantearlo como una propuesta a futuro, para conocer un poco más sobre el impacto de las TIC en los procesos de aprendizaje.

Por último, insistimos en la importancia que presenta el conocimiento y utilización de estrategias de aprendizaje para favorecer un aprendizaje autorregulado, por lo que compartimos opinión con aquellos estudios que consideran que la formación en el uso de estrategias de aprendizaje debiera considerarse dentro de los planes de estudio como aprendizajes transversales, ya que se ha comprobado que la puesta en práctica de estas herramientas favorece el rendimiento académico (Rosario, Fuentes, Beuchat y Ramaciotti, 2016).

Referencias

Boekaerts, M., \& Niemivirta, M. (2000). Self-regulated learning: finding a balance between learning goals and ego-protective goals. En M. Boekaerts, P. Pintrich, \& M. Zeidner, Handbook of Self-regulation (pp. 417-450). San Diego, California: Academic Press.

Borkowski, J., Chan, L., \& Muthukrishna, N. (2000). A processoriented model of metacognition: links between motivation and executive functioning. En G. Schraw, \& J. Impara, Issues in the Measurement of Metacognition (pp. 1-43). Lincoln, NE: Buros Institute of Mental Measurements Universidad de Nebraska.

Buendia, L., Colás, P. y Hernández, F. (1998). Métodos de investigación en Psicopedagogía. Madrid: McGraw-Hill.

Byrne, B. (2001). Structural equation modeling with Amos: Basic concepts, applications and programming. Mahwah, $\mathrm{NJ}$ : Erlbaum.
Coll, C., \& Colbs. (2002). El constructivismo en el aula. Barcelona: Graó.

Cubillo, M. (15 de 12 de 2010). EconPapers. Obtenido de http://repec.economicsofeducation.com/2010zaragoza/0558.pdf

De la Fuente, J. y Justicia, F. (2007). El modelo DIDEPRO de regulación de la enseñanza y del aprendizaje: Avances recientes. Revista electrónica de investigación psicoeducativa, 5(3), 535-564

Decreto 72/2003, de 18 de marzo, de Medidas de Impulso de la Sociedad del Conocimiento en Andalucía.

Gaeta, M.L. y Herrero, M.L. (2009). Influencia de las estrategias volitivas en la autorregulación del aprendizaje. Estudios de Psicología, 30(1), 73-88. 
González Pérez, A. y De Pablos Pons, J. (2015). Factores que dificultan la integración de las TIC en las aulas. Revista de investigación educativa, 33(2), 401-417.

Marco, B. (2008). Competencias básicas. Madrid: Narcea.

Maturana, H. y Varela, F. (2003). El árbol del conocimiento: Las bases biológicas del entendimiento humano. Buenos Aires: Lumen.

Pintrich, P. (2000). The role of goal orientation in self-regulated learning. En M. Boekaerts, P. Pintrich, \& M. Zeidner, Handbook of self-regulation (pp. 451-502). San Diego, California: Academic Press.

Rosario, P., Fuentes, S., Beuchat, M. y Ramaciotti, A. (2016). Autorregulación del aprendizaje en una clase de la universidad: un enfoque de difusión curricular. Revista de Investigación Educativa, 34(1), 31-49.
Vallejo, M., Fernández-Cano, A. y Torralbo, M. (2006). Patrones de citación en la investigación española en educación matemática. Revista española de documentación científica, 29(3), 382-397

Walber, M. (2005). Konstruktivismus als Legitimation für selbstgesteuertes Lernen mit neue Medien?! Report, 28(1), 102-109.

Werlen, E., \& Bergamin, P. (2012). Lebens - Langes Lernen. http://goo.gl/Oh3nTF

Winne, P., \& Perry, N. (2000). Measuring self-regulated learning. En P. Pintrich, M. Boekaerts, \& M. Zeidner, Handbook of selfregulation (pp. 531-566). Orlando, FL: Academic Press.

Zimmerman, B. (2000). Attaining self-regulation: A social cognitive perspective. En P. Pintrich, M. Boekaerts, \& M. Zeidner, Handbook of self-regulation (pp. 13-41). Orlando, FL: Academic Press.

\section{Revisión por pares abierta | Open Peer Review}

Dr. Miguel Ángel Gallardo Vigil. Universidad de Granada (Spain)

iD $\quad 0000-0002-5462-077 X$

Dra. Karen McMullin. Trent University (Canada)

(iD $\quad 0000-0002-1449-3550$ 